\title{
A Survey of Tables of Probability Distributions
}

\begin{tabular}{lll}
\hline Volume 110 & \multicolumn{1}{c}{ Number 1 } & January-February 2005 \\
\hline $\begin{array}{l}\text { Raghu Kacker } \\
\text { National Institute of Standards } \\
\text { and Technology, }\end{array}$ & $\begin{array}{l}\text { This article is a survey of the tables of } \\
\text { probability distributions published about } \\
\text { or after the publication in 1964 of the }\end{array}$ & $\begin{array}{l}\text { Key words: continuous univariate } \\
\text { distributions; discrete univariate } \\
\text { distributions; multivariate distributions; } \\
\text { probability distributions. }\end{array}$ \\
and & $\begin{array}{l}\text { Handbook of Mathematical Functions, } \\
\text { edited by Abramowitz and Stegun }\end{array}$ & Accepted: January 19, 2005 \\
Ingram Olkin & & \\
$\begin{array}{l}\text { Stanford University, } \\
\text { Stanford, CA 94305-4065 }\end{array}$ & & Available online: http://www.nist.gov/jres \\
raghu.kacker@nist.gov & & \\
\hline
\end{tabular}

\section{Introduction}

Probabilities and percentiles of statistical probability distributions have historically been cited from reference tables published in books, journals, and other publications. Reference tables of probability distributions continued to be published from the 1920s through the 1980s and early 1990s. Some tables superceded their earlier counterparts. Abramowitz and Stegun [1] surveyed the tables published before 1964, and reproduced some of them. In particular, Abramowitz and Stegun [1] reproduced the tables of percentiles of chi-square, $t-$, and F-distributions from the 1954 edition of Pearson and Hartley [2]. Other collections of tables of probability distributions include Greenwood and Hartley [3] and Owen [4].

This article is a survey of the tables published about or after 1964. A few earlier tables are also mentioned when appropriate. Most of the tables abstracted in this article are referenced in Pearson and Hartley [2], Pearson and Hartley [5], Johnson, Kotz, and Kemp [6], Johnson, Kotz, and Balakrishnan [7], Johnson, Kotz, and Balakrishnan [8], Johnson, Kotz, and Balakrishnan
[9], and Kotz, Balakrishnan, and Johnson [10]. The abstracts presented here have been verified from the original sources, and in some cases corrections and additions were made. The next three sections contain the abstracts for discrete univariate, continuous univariate, and multivariate probability distributions.

A random variable is denoted by $X$, and $x$ denotes a particular value of $X$. The cumulative distribution function of $X$ is $F(x)=\operatorname{Pr}\{X \leq x\}$. The survival function of $X$ is $\bar{F}(x)=1-F(x)=\operatorname{Pr}\{\mathrm{X}>x\}$. For a discrete random variable $f(x)$ interpreted as $\operatorname{Pr}\{X=x\}$ is the probability mass function (pmf). For a continuous random variable $f(x)$ interpreted as $\mathrm{d} F(x) / \mathrm{d} x$ is the probability density function (pdf). A particular value, $x$, is the rth quantile of $X$ when $F(x)=r$, for $0 \leq r \leq 1$. The rth quantile is commonly referred to as the $r \times 100$ th percentile of $X$. The expected value or mean, and the variance of $X$ are denoted by $E(X)$, and $V(X)$, respectively. The abbreviation $n \mathrm{D}$, for an integer $n$, denotes $n$ decimal places. An expression such as $0.01(0.02) 0.09$ denotes the sequence of numbers from 0.01 to 0.09 increasing in steps of 0.02. Log denotes natural logs unless indicated otherwise. 


\section{Discrete Univariate Distributions}

\subsection{Binomial Distribution}

The pmf is

$$
f(x)=\left(\begin{array}{l}
n \\
x
\end{array}\right) p^{x}(1-p)^{n-x}
$$

for $x=0,1, \ldots, n$.

Weintraub [11] tabulated, to $10 \mathrm{D}, \bar{F}(x-1)$ for $p=0.0001(0.0001) 0.0009,0.001(0.001) 0.1$, and $n=$ 1(1) 100 .

Pearson and Hartley [2] tabulated, to 5D, $f(x)$ for $p=0.01,0.02(0.02) 0.10,0.10(0.10) 0.50$, and $n=$ $5(5) 30$.

\subsection{Poisson Distribution}

The pmf is

$$
f(x)=\frac{\mathrm{e}^{-\theta} \theta^{x}}{x !}
$$

for $x=0,1, \ldots$.

Defense Systems Department, General Electric Company [12] tabulated, to $8 \mathrm{D}, f(x), F(x)$, and $\bar{F}(x-1)$ for $\theta$ ranging from $10^{-7}$ to 205 with increments ranging from $10^{-7}$ to 5 .

Khamis and Rudert [13] tabulated, to $10 \mathrm{D}, \bar{F}(x-1)$ for $\theta=0.00005(0.00005) 0.0005,0.0005(0.0005) 0.005$, $0.005(0.005) 0.5,0.5(0.025) 3,3(0.05) 8,8(0.25) 33$, $33(0.5) 83$, and $83(1) 125$.

Pearson and Hartley [2] tabulated, to 6D, $f(x)$ for $\theta=0.1(0.1) 15.0$. They also tabulated, to $5 \mathrm{D}$, $F(x)$ for $\theta=0.0005(0.0005) 0.005,0.005(0.005) 0.05$, $0.05(0.05) 1,1(0.1) 5,5(0.25) 10,10(0.5) 20,20(1) 60$, and $x=1(1) 35$.

\subsection{Negative Binomial Distribution}

The pmf is

$$
f(x)=\left(\begin{array}{c}
k+x-1 \\
k-1
\end{array}\right) p^{k}(1-p)^{x}
$$

for $\quad x=0, \quad 1, \quad \ldots \quad$ and $E(X)=k(1-p) / p$. Grimm [14] tabulated, to 5D, $f(x)$ and $F(x)$ for $E(X)=0.1(0.1) 1.0,1.0(0.2) 4.0,4.0(0.5) 10.0$, and $1 / p=1.2,1.5,2.0(1) 5$.

Williamson and Bretherton [15] tabulated, to 6D, $f(x)$ and $F(x)$ for the following values of $p$ and $k$ : $p=0.05$ and $k=0.1(0.1) 0.5, p=0.10$ and $k=0.1$ (0.1)1.0, $p=0.12(0.02) 0.20$ and $k=0.1(0.1) 2.5$, $p=0.22(0.02) 0.40$ and $k=0.1(0.1) 2.5(0.5) 5.0, p=0.42$ (0.02) 0.60 and $k=0.1(0.1) 2.5(0.5) 10.0, \quad p=0.62$ (0.02) $0.80 \quad$ and $k=0.2(0.2) 5.0(1) 20, \quad p=0.82$ (0.02)0.90 and $k=0.5(0.5) 10.0(2) 50, \quad p=0.95$ and $k=2(2) 50(10) 200$. Deahl [16] extended the Williamson and Bretherton [15] table of $F(x)$ for $p=0.02,0.04,0.05,0.06,0.08,0.10$, and $k=0.10$ (0.10)2.00.

Brown [17] tabulated, to 4D, $f(x)$ and $F(x)$ for $E(X)=0.25(0.25) 1.0,1.0(1) 10$, and $1 / p=1.5$ (0.5)5.0, 5(1)7.

\subsection{Hypergeometric Distribution}

The pmf is

$$
f(x)=\left(\begin{array}{l}
k \\
x
\end{array}\right)\left(\begin{array}{c}
N-k \\
n-x
\end{array}\right) /\left(\begin{array}{l}
N \\
n
\end{array}\right)
$$

for $\max [0, n-N+k] \leq x \leq \min [k, n]$.

Lieberman and Owen [18] tabulated, to 6D, $f(x)$ and $F(x)$ for $N=2(1) 100, n=1(1) 50$, and all possible values of $k ; N=1000, n=500$, and all possible values of $k$; and $N=100(100) 2000, n=N / 2$, and $k=(n-1)$.

\subsection{Logarithmic Series Distribution}

The pmf is

$$
f(x)=\frac{-\theta^{x}}{[\log (1-\theta)] x}
$$

for $x=1,2, \ldots$ and $E(X)=-\theta /[(1-\theta) \log (1-\theta)]$.

Patil [19] tabulated, to $4 \mathrm{D}, E(X)$ as a function of $\theta$ for $\theta=0.01(0.01) 0.99$. Patil, Kamat, and Wani [20] tabulated, to 6D, $f(x)$ and $F(x)$ for $\theta=0.01(0.01) 0.70$, $0.70(0.005) 0.900$, and 0.900(0.001)0.999. Patil and Wani [21] tabulated, to 4D, parameter $\theta$ for $E(X)=1.02(0.02) 2.00,2.00(0.05) 4.00$, and 4.00(0.1)8.0, $8.0(0.2) 16.0, \quad 16.0(0.5) 30.0, \quad 30.0(2) 40, \quad 40(5) 60$, 60(10)140, and 140(20)200.

Williamson and Bretherton [22] tabulated, to 5D, $f(x)$ and $F(x)$ for $E(X)=1.1(0.1) 2.0,2.0(0.5) 5.0$, $5.0(1) 10.0$. They also tabulated $\theta$, to $5 \mathrm{D}$, for $E(X)=$ 1.0(0.1)10.0, and 10.0(1)50.

\subsection{Neyman Type A Distribution}

This is the Poisson-stopped-summed-Poisson distribution (Johnson, Kotz, and Kemp [6]). The pmf is

$$
f(x)=\frac{\mathrm{e}^{-\lambda} \phi^{x}}{x !} \sum_{j=0}^{\infty} \frac{\left(\lambda \mathrm{e}^{-\phi}\right)^{j} j^{x}}{j !}
$$

for $x=0,1, \ldots$ and $E(X)=\lambda \phi$.

Grimm [23] tabulated, to 5D, $f(x)$ for $E(X)=$ $0.1(0.1) 1.0,1.0(0.2) 4,6,10$, and $\phi=0.2,0.5,1.0,2$, 3,4 up to $f(x)=0.99900$. 


\subsection{Geometric-Poisson Distribution}

The pmf is

$$
f(x)=\mathrm{e}^{-\theta} p^{x} \sum_{j=1}^{x}\left(\begin{array}{c}
x-1 \\
j-1
\end{array}\right) \frac{(\theta(1-p) / p)^{j}}{j !}
$$

for $x=1,2, \ldots ; f(x)=\mathrm{e}^{-\theta}$ for $x=0$; and $E(\mathrm{X})=$ $\theta /(1-p)$. This distribution is also called Pólya-Aeppli distribution.

Sherbrooke [24] tabulated, to 4D, $f(x)$ and $F(x)$ for $E(X)=0.10,0.25(0.25) 1.00,1.00(0.5) 3.0,3.0(1) 10$, and $(1+p) /(1-p)=1.5(0.5) 5.0,5.0(1) 7$.

\section{Continuous Univariate Distributions}

\subsection{Standard Normal (Gaussian) Distribution}

The pdf is

$$
f(x)=\frac{1}{\sqrt{2 \pi}} \mathrm{e}^{-x^{2} / 2} .
$$

Abramowitz and Stegun [1] tabulated the following: $F(x)$, to $15 \mathrm{D}$, for $x=0.00(0.02) 3.00$ and, to $10 \mathrm{D}$, for $x=3.00(0.05) 5.00 ; f(x)$, to 5D, for $x$ such that $\bar{F}(x)=q$ for $q=0.000(0.001) 0.500$; and $x$ such that $\bar{F}(x)=q$ for $q=0.000(0.001) 0.500,0.0000(0.0001)$ 0.0250 , and $q=10^{-m}$ for $m=4(1) 23$. They also tabulated the derivatives of $f(x)$ up to the order 12 .

Pearson and Hartley [5] tabulated, to 10D, quantiles $x$ and corresponding $f(x)$, where $F(x)=p$ for $p=0.500(0.001) 0.999$, and 0.9990(0.0001)0.9999.

White [25] tabulated, to 20D, quantiles $x$ such that $\bar{F}(x)=q$ for $q=0.005(0.005) 0.500$, and $q=5 \times 10^{-k}$, $2.5 \times 10^{-k}$, and $1 \times 10^{-k}$, where $k=1(1) 20$.

\subsection{Standardized Stable Distributions}

The pdfs of standardized stable distributions are unimodal with shape depending on the parameters $\beta$ and $\alpha$. Although the pdfs are rather complicated, they can be expressed as convergent series (Johnson, Kotz, and Balakrishnan [7]).

Fama and Roll [26] tabulated, to 4D, $F(x)$ for $\beta=0$ and $\alpha=1.0(0.1) 1.9,1.95,2.0$, and $x=$ $0.05(0.05) 1.00,1.00(0.1) 2.0,2.0(0.2) 4.0,4.0(0.4) 6.0$, $6.0(1) 8,10,15$, and 20. They also tabulated, to $3 \mathrm{D}$, quantiles $x$ such that $F(x)=p$, for $p=$ $0.52(0.02) 0.94,0.94(0.01) 0.97,0.97(0.005) 0.995$, and 0.9975 .

Holt and Crow [27] tabulated, to 4D, $f(x)$ for $\beta=-1.00(0.25) 1.00$ and $\alpha=0.25(0.25) 2.00$, and nonnegative $x$ in steps varying by factors of 10 from 0.001 to 100 such that interpolation is possible. The tabulation is terminated when $f(x)$ first falls to 0.0001 . The largest such value of $x$ is 338 , for $\alpha=0.25$ and $\beta=-1.00$.

Worsdale [28] tabulated, to 4D, $F(x)$ for $\beta=0$ and $\alpha=0.6(0.1) 2.0$, and $x=0.00(0.05) 3.00$. For larger values of $x, F(x)$ is tabulated for $\log _{10} x=$ $0.40(0.05) 2.50$.

Panton [29] tabulated, to 5D, $F(x)$ for $\beta=0$ and $\alpha=1.0(0.1) 2.0$, and $x=0.05(0.05) 1.00,1.00(0.1) 2.0$, $2.0(0.2) 4.00,4.00(0.4) 6.0,7,8,10,15,20$

\subsection{Inverse Gaussian Distribution}

The pdf is

$$
f(x)=\left(\frac{\lambda}{2 \pi x^{3}}\right)^{1 / 2} \exp \left(\frac{-\lambda(x-\mu)^{2}}{2 \mu^{2} x}\right)
$$

for $x>0, \lambda>0, \mu>0$, and $E(X)=\mu$ and $V(X)=$ $\mu^{3} / \lambda$.

Wasan and Roy [30] tabulated, to 4D, quantiles $x$ such that $\mu=t, \lambda=t^{2}$, that is, $\mu=t=V(X)$, and $F(x)=p$, where $t=0.1(0.1) 4.0,4.0(0.2) 6.0$, $6.0(1.0) 35.0, \quad 35(5) 100,100(10) 150, \quad 150(20) 250$, 300(100)1000, 1000(200)1600, 2000(400)4000, and $p=0.005,0.010,0.025(0.025) 0.100,0.25(0.25) 0.75$, $0.80,0.900(0.025) 0.975$, and 0.990 . In order to determine quantiles of an inverse Gaussian random variable $Y$ with parameters $\mu>0$ and $\lambda>0$, use the fact that the distribution of $X=\lambda Y / \mu^{2}$ is inverse Gaussian with parameters $t$ and $t^{2}$, where $t=\lambda / \mu$.

Chan, Cohen, and Whitten [31] tabulated $F(x)$ of the standardized inverse Gaussian distribution with $E(X)=0$ and $V(X)=1$ for various values of the standardized third moment about the mean $\alpha_{3}=\sqrt{\beta}_{1}^{-}$. They tabulated, to $6 \mathrm{D}, F(x)$ for $x=$ $-3.0(0.1) 5.9$ with $\alpha_{3}=0.0(0.1) 1.2$, and $x=-1.5(0.1) 7.4$ with $\alpha_{3}=1.3(0.1) 2.5$.

Koziol [32] tabulated quantiles $x$, to eight significant digits, such that $F(x)=p, \mu=t, \lambda=t^{2}$, where $t=$ $0.02(0.02) 4,4(0.04) 6,6(0.02) 35,35(1) 100,100(2) 150$, $150(4) 250,250(10) 300,300(20) 600,600(40) 2000$, $2000(80) 4000$, and $p=0.001,0.005,0.01(0.01) 0.99$, $0.995,0.999$.

\subsection{Incomplete Gamma Function}

Harter [33] tabulated, to 9D, the incomplete $\Gamma$-function ratio $I(u, p)$ defined by Pearson [34] as

$$
I(u, p)=\frac{1}{\Gamma(p+1)} \int_{0}^{u \sqrt{p+1}} v^{p} \mathrm{e}^{-v} \mathrm{~d} v
$$


for $u$ at intervals of 0.1 , starting from 0.0 , and $p=-0.5(0.5) 74$ and 74(1)164. Harter [35] extended Harter [33] for $p=-0.95(0.05) 4$.

\subsection{Standard Gamma Distribution}

The pdf is

$$
f(x)=\frac{1}{\Gamma(\alpha)} x^{\alpha-1} \mathrm{e}^{-x}
$$

for $x \geq 0$ and $\alpha>0$.

Wilk, Gnanadesikan, and Huyett [36] tabulated quantiles $x$, accurate to about five significant digits, for $\quad \alpha=0.1(0.1) 0.6, \quad 0.6(0.2) 5.0, \quad 5.0(0.5) 10.0$, $10.0(1.0) 20.0$, and $p=0.1,0.5,0.7,1.0(0.5) 3.0$, $3.0(1.0) 5.0,7.5,10.0(5.0) 30.0,30(10) 70,70(5) 90$, $90.0(2.5) 97.5,98.0,99.0,99.5$, and 99.9 .

Thom [37] tabulated, to 4D, $F(x)$ for $\alpha=$ $0.5(0.5) 15.0, \quad 15(1) 36$, and $x=0.0001,0.001$, $0.004(0.002) 0.020, \quad 0.02(0.02) 0.80, \quad 0.8(0.1) 2.0$, 2.0(0.2)3.0, 3.0(0.5)9.0; also tabulated, to $4 \mathrm{D}$, quantiles $x$ and corresponding $f(x)$ such that $F(x)=p$ for $\alpha=$ $0.5(0.5) 15.0,15(1) 36$, and $p=0.01,0.05(0.05) 0.95$, 0.99 .

Harter [38] tabulated, to 5D, quantiles $x$ such that $F(x)=p$ against the coefficient of skewness ${\sqrt{\beta_{1}}}^{-}=\mu_{3} / \sigma^{3}=2 / \sqrt{\alpha}$ for ${\sqrt{\beta_{1}}}^{-}=0.0(0.1) 4.8$, and 4.8(0.2)9.0, and $p=0.0001,0.0005,0.0010,0.0050$, $0.0100, \quad 0.0200, \quad 0.0250, \quad 0.0400, \quad 0.0500$, $0.1000(0.1000) 0.9000,0.9500,0.9600,0.9750,0.9800$, $0.9900,0.9950,0.9990,0.9995$ and 0.9999 . Harter [39] extended Harter [38] for $p=0.002000,0.429624$, 0.570376 , and 0.998000 .

\subsection{Chi-Square Distribution}

The pdf is

$$
f(x)=x^{(v-2) / 2} /\left[2^{v / 2} \Gamma(v / 2) \mathrm{e}^{-x / 2}\right]
$$

for $x>0$ and degrees of freedom $v>0$. If $X_{1}, X_{2}$, $\ldots, X_{v}$ have independent standard normal distributions, then $X=\sum_{i=1}^{n} X_{i}^{2}$ has a chi-square distribution with $v$ degrees of freedom.

Harter [33] tabulated, to six significant digits, quantiles $x$ such that $F(x)=p$ for $p=0.0001,0.0005$, $0.001,0.005,0.01,0.025,0.05,0.1(0.1) 0.9,0.95$, $0.975,0.99,0.995,0.999,0.9995$, and 0.9999 , and $v=1(1) 150$, and 150(2)330. A subset of these tables for $v=1(1) 100$ is reproduced in Harter [40].

Khamis and Rudert [13] tabulated $F(x)$, to 10D, for $v=0.1(0.1) 20,20(0.2) 40,40(0.5) 140$, and $x=$ $0.0001(0.0001) 0.001,0.001(0.001) 0.01,0.01(0.01) 1$, $1(0.05) 6,6(0.1) 16,16(0.5) 66,66(1) 166,166(2) 250$.
Pearson and Hartley [5] tabulated, to six significant digits, quantiles $x$ such that $F(x)=p$ for $p=0.0001$, $0.0005,0.001,0.005,0.01,0.025,0.05,0.1(0.1) 0.9$, $0.95,0.975,0.99,0.995,0.999,0.9995$, and 0.9999 , and $v=0.1(0.1) 3.0,3.0(0.2) 10.0$, and 10(1)100.

Mardia and Zemroch [41] tabulated, to five significant digits, quantiles $x$ such that $F(x)=p$ for $p=0.0001$, $0.0005,0.001,0.005,0.01,0.02,0.025,0.03(0.01) 0.1$, $0.2,0.25,0.3(0.1) 0.7,0.75 .0 .8,0.9(0.01) .97,0.975$, $0.98,0.99,0.995,0.999,0.9995,0.9999$, and fractional degrees of freedom $v=0.1(0.1) 3.0,3.0(0.2) 7.0$, $7.0(0.5) 11,11(1) 30,30(5) 60,60(10) 120$.

\subsection{Standardized Weibull Distribution}

The pdf is

$$
f(x)=\gamma x^{\gamma-1} \mathrm{e}^{-x^{\gamma}}
$$

for $x>0$ and $\gamma>0$, where $\gamma$ is the shape parameter.

Plait [42] tabulated, to $8 \mathrm{D}, f(x)$ for $\gamma=0.1(0.1) 3$, 3(1)10, and tabulated, to 7D, $F(x)$ for $\gamma=0.1(0.1) 4.0$.

\subsection{Standardized Extreme Value Distribution -Type 1}

The pdf is

$$
f(x)=\exp \left(-x-\mathrm{e}^{-x}\right)
$$

Gumbel [43] tabulated, to 7D, $f(x)$ and $F(x)$ for the following values of $x$ : $-3.0(0.1)-2.4$, $-2.40(0.05) 0.00, \quad 0.0(0.1) 4.0, \quad 4.0(0.2) 8.0, \quad$ and 8.0(0.5)17.0. Also, tabulated, to 5D, quantiles $x$ such that $F(x)=p$ for $p=0.0001(0.0001) 0.0050$, $0.005(0.001) 0.988, \quad 0.9880(0.0001) 0.9994, \quad$ and $0.99940(0.00001) 0.99999$.

White [44] tabulated, to 7D, the means and variances of all order statistics for sample sizes 1(1)50 and 50(5)100. Extended tables of means, variances, and covariances of all order statistics for sample sizes up to 30 have been provided by Balakrishnan and Chan [45] and Balakrishnan and Chan [46].

\subsection{Incomplete Beta Function}

Pearson [47] tabulated, to 7D, the incomplete $B$-function ratio $I(p, q)$ defined as

$$
I(p, q)=\frac{\Gamma(p+q)}{\Gamma(p) \Gamma(q)} \int_{0}^{x} t^{p-1}(1-t)^{q-1} \mathrm{~d} t
$$

for $p, q=0.5(0.5) 11.0(1) 50$ with $p \geq q$ and $x=$ $0.00(0.01) 1.00$. These values are reproduced in Pearson [48]. Additional values of $I(p, q)$ are given, to $7 \mathrm{D}$, for 
$p=11.5(1.0) 14.5, q=0.5$, and $x=0.00(0.01) 1.00$. More values of $I(p, q)$ are given, to $7 \mathrm{D}$, for $p=0.5(0.5) 11.0(1) 16, q=1.0(0.5) 3.0$, and $x=0.975$, $0.980,0.985,0.988(0.001) 0.999$. Even more values of $I(p, q)$ are given for $p=0.5(0.5) 11.0(1) 16, q=0.5$, and $x=0.9750,0.9800,0.9850,0.9880(0.0005) 0.9985$, $0.9988(0.0001) 0.9999$. For $x \geq 0.988$, values are given to $8 \mathrm{D}$

\subsection{Beta Distribution}

The pdf is

$$
f(x)=\frac{1}{B(a, b)} x^{a-1}(1-x)^{b-1}
$$

for $\quad 0<x<1, \quad a>0, \quad b>0 \quad$ and $\quad B(a, b)=$ $\Gamma(a) \Gamma(b) / \Gamma(a+b)$.

Harter [33] tabulated, to 7D, quantiles $x$ such that $F(x)=p$ for $a=1(1) 40, b=1(1) 40$, and $p=0.0001$, $0.0005,0.001,0.005,0.01,0.025,0.05,0.1(0.1) 0.9$, $0.95,0.975,0.99,0.995,0.999,0.9995,0.9999$.

Vogler [49] tabulated, to six significant digits, quantiles $x$ such that $F(x)=p$ for $a=0.50(0.05) 1.00,1.1$, $1.25(0.25) 2.50,2.50(0.5) 5.0,6,7.5,10,12,15,20,30,60$, $b=0.5(0.5) 5.0,6,7.5,10,12,15,20,30,60$, and $p=0.0001,0.001,0.005,0.01,0.025,0.05,0.1,0.25,0.5$.

Pearson and Hartley [2] tabulated, to five significant digits, quantiles $x$ such that $F(x)=p$ for $a=0.5(0.5) 15.0,20,30,60, b=0.5(0.5) 5.0,6,7.5,10$, $12,15,20,30,60$, and $p=0.001,0.0025,0.005$, $0.01,0.025,0.05,0.10,0.25,0.50$.

\subsection{F-Distribution}

If $X_{1}$ and $X_{2}$ have independent chi-square distributions with degrees of freedom $v_{1}$ and $v_{2}$, respectively, then

$$
X=\frac{X_{1} / v_{1}}{X_{2} / v_{2}}
$$

has an F-distribution with $v_{1}$ (numerator) and $v_{2}$ (denominator) degrees of freedom.

Pearson and Hartley [5] tabulated, to five significant digits, quantiles $x$ such that $F(x)=p$ for $p=0.5$, $0.75,0.90,0.95,0.975,0.99,0.995,0.9975,0.999$, and $v_{1}=1(1) 10,12,15,20,24,30,40,60,120, \infty$, and $v_{2}=1(1) 30,40,60,120, \infty$.

Mardia and Zemroch [41] tabulated, to five significant digits, quantiles $x$ such that $F(x)=p$ for $p=0.5,0.6,0.7,0.75,0.8,0.90(0.01) 0.99,0.975$, $0.995,0.999,0.9995,0.9999$, and $v_{1}=0.1(0.1) 1.0$, $1.0(0.2) 2.0,2.0(0.5) 5,5(1) 16,18,20,24,30,40,60$, $120, \infty$, and $v_{2}=0.1(0.1) 3.0,3.0(0.2) 7.0,7.0(0.5) 11$,
$11(1) 40,60,120, \infty$. A part of this table is reproduced in Pearson and Hartley [5].

\subsection{2 t-Distribution}

If $X_{1}$ has the standard normal distribution and $X_{2}$ has an independent chi-square distribution with $v$ degrees of freedom, then

$$
X=X_{1} / \sqrt{X_{2} / v}
$$

has a Student's t-distributon with $v$ degrees of freedom.

Fisher and Yates [50] tabulated, to 3D, quantiles $x$ such that $F(x)=p$ for $p=0.55(0.05) 0.95,0.975$, $0.99,0.995,0.9995$, and $v=1(1) 30,40,60,120$. Lempers and Louter [51] extend these tables for $p=0.5625(0.0625) 0.9375$.

Hill [52] tabulated, to 20D or 20 significant digits, quantiles $x$ such that $F(x)=p / 2$ where $p=0.9(-0.1) 0.1$, $10^{-m}, 2 \times 10^{-m}, 5 \times 10^{-m}$, for $m=2(1) 10(5) 30$, and $v=1(1) 30,30(2) 50,50(5) 100,100(10) 150,200,[240$, $300,400,600,1200] \times[1,10,100]$, and $\infty$.

Mardia and Zemroch [41] tabulated, to five significant digits, quantiles $x$ such that $F(x)=p$ for $p=0.5$, $0.6,0.7,0.75,0.8,0.90(0.01) 0.99,0.975,0.995,0.999$, $0.9995,0.9999$, and fractional degrees of freedom $v=0.1(0.1) 3.0,3.0(0.2) 7.0,7.0(0.5) 11,11(1) 40,60$, 120 , and $\infty$.

\subsection{Noncentral Chi-Square Distribution}

If $X_{1}, X_{2}, \cdots, X_{v}$ have independent standard normal distributions and $\delta_{1}, \delta_{2}, \cdots, \delta_{v}$ are constants then

$$
X=\sum_{i=1}^{v}\left(X_{i}+\delta_{i}\right)^{2}
$$

has a noncentral chi-square distribution with $v$ degrees of freedom and noncentrality parameter $\lambda=\Sigma_{i=1}^{v} \delta_{i}^{2}$.

Johnson [53] tabulated, to four significant digits, quantiles $x$ such that $F(x)=p$ for $p=0.001,0.0025$, $0.005,0.01,0.025,0.05,0.10,0.25,0.5,0.75,0.90$, $0.95,0.975,0.99,0.995,0.9975,0.999, v=1(1) 12$, 15,20 , and square root of the noncentrality parameter $\sqrt{\lambda}=0.2(0.2) 6.0$.

Haynam, Govindarajulu, and Leone [54] tabulated the power $1-\beta$ of chi-square test of significance as a function of the level of significance $\alpha$, degrees of freedom $v$, and noncentrality parameter $\lambda$ for $\alpha=0.001,0.005,0.01,0.025,0.05,0.1, v=1(1) 30$, $30(2) 50,50(5) 100$, and $\lambda=0.0(0.1) 1.0,1.0(0.2) 3.0$, $3.0(0.5) 5.0,5(1) 40,40(2) 50,50(5) 100$. They also tabulated the noncentrality parameter $\lambda$ as a function of $\alpha, v$, and $1-\beta$ for the values of $\alpha$ and $v$ listed above and $1-\beta=0.1(0.02) 0.7,0.7(0.01) 0.99$. 
Pearson and Hartley [5] tabulated, to 3D, noncentrality parameter $\lambda$ as a function of the level of significance $\alpha$, degrees of freedom $v$, and power $1-\beta$ for $\alpha=0.05,0.01, \quad v=1(1) 30,30(2) 50,50(5) 100$, and $1-\beta=0.25,0.50,0.60,0.70(0.05) 0.95,0.97,0.99$.

\subsection{Noncentral Chi Distribution}

If $X_{1}$ has a noncentral chi-square distributon then the distribution of $X=\sqrt{X_{1}}$ is referred to as noncentral chi distribution.

Johnson and Pearson [55] tabulated, to four significant digits, quantiles $x$ of chi distribution such that $F(x)=p$ for $p=0.005,0.01,0.025,0.05,0.95,0.975$, $0.99,0.995$, degrees of freedom $v=1(1) 12,15,20$, and square root of the noncentrality parameter $\sqrt{\lambda}=$ $0.0(0.2) 6.0$. Approximate quantiles to three significant digits are also given for $\sqrt{\lambda}=8.0$ and 10.0. These tables are reproduced in Pearson and Hartley [5].

\subsection{Noncentral F-Distribution}

If $X_{1}$ has a noncentral chi-square distribution with $v_{1}$ degrees of freedom and noncentrality parameter $\lambda$, $X_{2}$ has a chi-square distribution with $v_{2}$ degrees of freedom, and $X_{1}$ and $X_{2}$ are independently distributed then

$$
X=\frac{X_{1} / v_{1}}{X_{2} / v_{2}}
$$

has a noncentral F-distribution with $v_{1}$ and $v_{2}$ degrees of freedom and noncentrality parameter $\lambda$.

Tiku [56] tabulated, to $4 \mathrm{D}$, the power of the F-test for the level of significance $\alpha=0.005,0.01,0.025$, $0.05, v_{1}=1(1) 10,12$, and $v_{2}=2(2) 30,40,0,120, \infty$, and noncentrality parameter $\lambda$ such that $\sqrt{\lambda /\left(v_{1}+1\right)}=$ $0.5,1.0(0.2) 2.2,2.2(0.4) 3.0$.

\subsection{Doubly Noncentral F-Distribution}

If $X_{1}$ has a noncentral chi-square distribution with $v_{1}$ degrees of freedom and noncentrality parameter $\lambda_{1}$, $X_{2}$ has a noncentral chi-square distribution with $v_{2}$ degrees of freedom and noncentrality parameter $\lambda_{2}$, and $X_{1}$ and $X_{2}$ are independently distributed then

$$
X=\frac{X_{1} / v_{1}}{X_{2} / v_{2}}
$$

has a doubly noncentral F-distribution with $v_{1}$ and $v_{2}$ degrees of freedom, and noncentrality parameters $\lambda_{1}$ and $\lambda_{2}$.

Tiku [57] tabulated, to $4 \mathrm{D}$, the power of the F-test for the level of significance $\alpha=0.01$ and 0.05 , degrees of freedom $v_{1}=1(1) 8,10,12,24$ and $v_{2}=2(2) 12,16$,
$20,24,30,40,60$, noncentrality parameters $\lambda_{1}$ and $\lambda_{2}$ such that $\phi_{1}=\sqrt{\lambda_{1} /\left(v_{1}+1\right)}=0(0.5) 3$ and $\phi_{2}=\lambda_{2} / \sqrt{v_{2}}$ $=0(1) 8$. Tiku [57] also tabulated, to $4 \mathrm{D}$, the power of F-test for the critical values $F_{0}$ such that $u_{0}=1 /[1+$ $\left.\left(v_{1} / v_{2}\right) F_{0}\right]=0.02(0.08) 0.50,0.60,0.75,0.95$, degrees of freedom $v_{1}=v_{2}=4(2) 12$, and the same noncentrality parameters as used in the previous table.

\subsection{Noncentral t-Distribution}

If $X_{1}$ has the standard normal distribution and $X_{2}$ has an independent chi-square distribution with $v$ degrees of freedom, then

$$
X=\left(X_{1}+\delta\right) / \sqrt{X_{2} / v}
$$

has a noncentral t-distributon with $v$ degrees of freedom and noncentrality parameter $\delta$.

Bagui [58] tabulated, to 5D, quantiles $x$ of noncentral t-distribution such that $F(x)=p$ for $p=0.01,0.025$, $0.05,0.10,0.20,0.30,0.70,0.80,0.90,0.95,0.975$, 0.99 , degrees of freedom $v=1(1) 60$, and noncentrality parameter $\delta=0.1(0.1) 8.0$.

\subsection{Doubly Noncentral t-Distribution}

If $X_{1}$ has the standard normal distribution and $X_{2}$ has an independent noncentral chi-square distribution with $v$ degrees of freedom and noncentrality parameter $\lambda$, then

$$
X=\left(X_{1}+\delta\right) / \sqrt{X_{2} / v}
$$

has a doubly noncentral t-distributon with $v$ degrees of freedom, numerator noncentrality parameter $\delta$, and denominator noncentrality parameter $\lambda$.

Bulgren [59] tabulated, to 6D, $F(x)$ of doubly noncentral t-distribution with degrees of freedom $v=$ 2(1)20, absolute value of numerator noncentrality parameter $|\delta|=0(1) 5$, denominator noncentrality parameter $\lambda=0,1,2(2) 8$, and $x=0.0,0.1,0.2(0.2) 9.0$.

\subsection{Distribution of the Sample Correlation Coefficient From Bivariate Normal Distribution}

Suppose $\left(Y_{i}, Z_{i}\right)$, for $i=1,2, \ldots, n$, are independently distributed and have a common joint bivariate normal distribution with correlation coefficient $\rho$. Then the sample correlation coefficient

$$
X=\frac{\sum_{i=1}^{n}\left(Y_{i}-\bar{Y}\right)\left(Z_{i}-\bar{Z}\right)}{\sqrt{\sum_{i=1}^{n}\left(Y_{i}-\bar{Y}\right)^{2} \sum_{i=1}^{n}\left(Z_{i}-\bar{Z}\right)^{2}}}
$$


has a distribution that depends only on the correlation coefficient $\rho$ and the sample size $n$.

Odeh [60] tabulated, to 5D, quantiles $x$ of the sample correlation coefficient, where $F(x)=p$ for $p=0.005,0.01,0.025,0.05,0.10,0.25,0.75,0.90,0.95$, $0.975,0.99,0.995, \rho=0.0(0.10) 0.90,0.95$, and $n=4(1) 30,30(2) 40,40(5) 50,50(10) 100,100(20) 200$, and 200(100)1000.

\subsection{Distribution of the Sample Multiple Correlation Coefficient From Multivariate Normal Distribution}

If the random variables $X_{1}, \ldots, X_{M}$ have a joint multivariate normal distribution, then the smallest mean squared error linear predictor of $X_{1}$ is the conditional expected value $E\left(X_{1} \mid x_{2}, \ldots, x_{M}\right)$. The multiple correlation coefficient $R$ is the correlation between $X_{1}$ and its smallest mean squared error linear predictor. The distribution of the sample multiple correlation coefficient $r$ depends only on the population coefficient $R$, number of variates $M$, and the sample size $N$.

Pearson and Hartley [5] tabulated, to 3D, lower and upper 1 and 5 percent points of the sample multiple correlation coefficient for $R=0.1(0.1) 0.9$, the sample size $N$ such that $N-M=10(10) 50$, and $M-1=2(2) 12,12(4) 24,30,34,40$.

\section{Multivariate Distributions}

\subsection{Multivariate Normal Distribution}

The multivariate normal density function of the random vector $\left(X_{1}, \ldots, X_{M}\right)$ is

$$
\begin{aligned}
& f\left(x_{1}, \ldots, x_{M}\right)= \\
& \frac{|\boldsymbol{\Sigma}|^{-1 / 2}}{(2 \pi)^{M / 2}} \exp \left[-(1 / 2)(\boldsymbol{x}-\boldsymbol{\mu})^{\prime} \boldsymbol{\Sigma}^{-1}(\boldsymbol{x}-\boldsymbol{\mu})\right],
\end{aligned}
$$

where $\boldsymbol{x}=\left(x_{1}, \ldots, x_{M}\right)^{\prime}, \boldsymbol{\mu}=\left(\mu_{1}, \ldots, \mu_{M}\right)^{\prime}$ is the mean vector, and $\boldsymbol{\Sigma}=\left[\sigma_{i j}\right]$ is the positive definite covariance matrix. Here $\left(x_{1}, \ldots, x_{M}\right)^{\prime}$, denotes transpose of the vector $\left(x_{1}, \ldots, x_{M}\right)$. For the case $\boldsymbol{\mu}=(0, \ldots, 0)^{\prime}, \sigma_{i i}=1, \sigma_{i j}=\rho$, where $0 \leq \rho<1$ and $i, j=1, \ldots, M, i \neq j$, a one-sided upper equicoordinate $p \times 100$ percentage point $g$ is such that

$$
\operatorname{Pr}\left\{\max _{1 \leq i \leq M} X_{i} \leq g\right\}=p,
$$

and a two-sided upper equicoordinate $p \times 100$ percentage point $h$ is such that

$$
\operatorname{Pr}\left\{\max _{1 \leq i \leq M}\left|X_{i}\right| \leq h\right\}=p,
$$

where $p$ is a specified value for the probability integral.

Gupta [61] tabulated equicoordinate one-sided probabilities $p$, to $5 \mathrm{D}$, for $g=-3.5(0.1) 3.5, M=1(1) 12$, and $\rho=0.100,0.125,0.200(0.05) 0.300,1 / 3,0.375$, $0.400(0.1) 0.600,0.625,2 / 3,0.700(0.05) 0.800,0.875$, and 0.900 .

Tong [62] tabulated equicoordinate one-sided and two-sided percentage points, to $4 \mathrm{D}$, and probability integrals $p$, to $5 \mathrm{D}$. The table of one-sided percentage points gives the values of $g$ for $M=2(1) 20$, $\rho=0.0(0.1) 0.9,1 / 3,2 / 3,1 / 4$, and $3 / 4$, and $p=0.90$, 0.95 , and 0.99 . The table of one-sided probability integrals gives the values of $p$ for $g=-2.0(0.1) 4.0, M=$ $2(1) 10,10(2) 20$, and $\rho=0.0(0.1) 0.9,1 / 3,2 / 3,1 / 4$, and $3 / 4$. The table of two-sided percentage points gives the values of $h$ for the same set of $M, \rho$, and $p$ as the onesided percentage points. The table of two-sided probability integrals gives the values of $p$ for $h=0.1(0.1) 5.0$ and the same set of $M$, and $\rho$ as the one-sided probability integrals.

\subsection{Multivariate t-Distribution}

Suppose the random vector $\boldsymbol{Z}=\left(Z_{1}, \ldots, Z_{M}\right)^{\prime}$ has a multivariate normal distribution with mean $\boldsymbol{\mu}=\mathbf{0}$ and covariance matrix $\boldsymbol{\Sigma}=\left[\sigma_{i j}\right]$, where $\sigma_{i i}=1$ for $i, j=1, \ldots, M$ (that is, $\boldsymbol{\Sigma}$ is a correlation matrix). Suppose $S$ is a random variable independent of $\boldsymbol{Z}$ such that $v S$ has a chi-square distribution with $v$ degrees of freedom. Then the joint distribution of $\left(T_{1}, \ldots, T_{M}\right)^{\prime}=\left(Z_{1} / S, \ldots, Z_{M} / S\right)^{\prime}$ is called a multivariate t-distribution with parameters $\boldsymbol{\Sigma}$ and $v$. A onesided upper equicoordinate $p \times 100$ percentage point $g$ is such that

$$
\operatorname{Pr}\left\{\max _{1 \leq i \leq M} T_{i} \leq g\right\}=p,
$$

and a two-sided upper equicoordinate $p \times 100$ percentage point $h$ is such that

$$
\operatorname{Pr}\left\{\max _{1 \leq i \leq M}\left|T_{i}\right| \leq h\right\}=p .
$$

Freeman, Kuzmack, and Maurice [63] tabulated percentage points $g$ to, $3 \mathrm{D}$, for $M=2$, and to, 2D, for $M=3,4,5$, for $p=0.95, v=(M+1) k$ for $k=9(10) 99,199,499$, and the following correlation structure: $\rho_{i j}=-1 / 2$ for $|i-j|=1$ and $\rho_{i j}=0$ for $|i-j|>1$, where $1 \leq i, j \leq M$. Freeman and Kuzmack [64] tabulated percentage points $g$, to $2 \mathrm{D}$, for the 
same correlation structure for $M=5(2) 9,9(5) 29, p=$ $0.90,0.95,0.99$ and $v=(M+1) k$ for $k=9,19,49,99$, 499, using Monte Carlo sampling.

Dunn, Kronmal, and Yee [65] computed, using Monte Carlo sampling, probabilities $\operatorname{Pr}\left\{\max _{1 \leq i \leq M}\left|T_{i}\right|\right.$ $\leq h\}=p$, to $4 \mathrm{D}$, for $M=2(2) 20, \rho=0.0(0.1) 0.9$, $h=0.2(0.2) 6.0$, and $v=4(2) 12,12(4) 24,30, \infty$.

For the bivariate case $M=2$, Krishnaiah, Armitage, and Breiter [66] tabulated probabilities $\operatorname{Pr}\left\{\max _{1 \leq i \leq M} T_{i} \leq g\right\}=p$, to $6 \mathrm{D}$, for $\pm \rho=0.0(0.1) 0.9$, $g=1.0(0.1) 5.5$, and $v=5(1) 35$. Also for $M=2$, Krishnaiah, Armitage, and Breiter [67] tabulated probabilities $\operatorname{Pr}\left\{\max _{1 \leq i \leq M}\left|T_{i}\right| \leq h\right\}=p$, to $6 \mathrm{D}$, for $|\rho|=0.0(0.1) 0.9, h=1.0(0.1) 5.5$, and $v=5(1) 35$.

Tong [68] tabulated percentage points $g$ for the following correlation structure: $\rho_{i j}=1$ for $i=j$, $\rho_{i j}=1 / 2$ for $i \neq j$ and $1 \leq i, j \leq m$ or $m<i, j \leq M$, $\rho_{i j}=-1 / 2$ for $1 \leq i \leq m$ and $m<j \leq M$ or $1 \leq j \leq m$ and $m<i \leq \mathrm{M}$ where $m=M / 2$ if $M$ is even and $m=(M+1) / 2$ if $M$ is odd. His Table 1 gives $g$, to $7 \mathrm{D}$, for $M=1(1) 10,10(2) 20, p=0.50,0.75,0.90,0.95$, $0.975,0.99$ and degrees of freedom $v=\infty$. His Table 2 gives $g$, to $5 \mathrm{D}$, for $M=2(1) 6,6(2) 12,12(4) 20$, $p=0.50,0.75,0.90,0.95,0.975,0.99$ and degrees of freedom $v=5(1) 10,10(2) 20,20(4) 60,60(30) 120$.

Trout and Chow [69] tabulated two-sided nonequicoordinate $p \times 100$ percentage points of trivariate $(M=3)$ t-distribution with non-singular correlation matrix

$$
\boldsymbol{\Sigma}=\left[\begin{array}{ccc}
1.0 & \rho_{12} & \rho_{13} \\
\rho_{12} & 1.0 & \rho_{23} \\
\rho_{13} & \rho_{23} & 1.0
\end{array}\right] .
$$

They tabulated $d$, to $2 \mathrm{D}$, where

$$
\int_{-d}^{d} \int_{-a d}^{a d} \int_{-b d}^{b d} f\left(t_{1}, t_{2}, t_{3} \mid \Sigma, v\right) \mathrm{d} t_{1} \mathrm{~d} t_{2} \mathrm{~d} t_{3}=p,
$$

for $p=0.95, \quad v=5(1) 9(2) 29, \quad a=0.5(0.1) 1.5, \quad b=$ $0.5(0.1) 1.5$, and a set of 22 triplets $\left(\rho_{12}, \rho_{13}, \rho_{23}\right)$, where $\rho_{i j}=0.0,0.1,0.5,0.9,(i \neq j, 1 \leq i, j \leq 3)$.

Dutt [70] tabulated the probabilities $\operatorname{Pr}\left\{\max _{1 \leq i \leq M} T_{i}\right.$ $\leq g\}=p$, to $6 \mathrm{D}$, for $g=0.0(0.5) 2.0,2.0(1.0) 4.0$, and $v=8(4) 40$, and $\infty$ : for $M=3$ with $\left(\rho_{12}, \rho_{13}, \rho_{23}\right)=(0.3$, $0.5,0.7)$, and $(0.1,0.3,0.5)$; and for $M=4$ with $\left(\rho_{12}\right.$, $\left.\rho_{13}, \rho_{14,} \rho_{23}, \rho_{24}, \rho_{34}\right)=(0.05,0.10,0.15,0.25,0.60,0.80)$, and $(0.25,0.35,0.50,0.60,0.65,0.70)$.

Bechhofer and Dunnett [71] tabulated one-sided and two-sided upper equicoordinate percentage points for $M=2(1) 16,16(2) 20$, degrees of freedom $v=2(1) 30,30(5) 50,60(20) 120,200, \infty$, and $\rho=$
$0.0(0.1) 0.9$, and $1 /(1+\sqrt{M})$. They tabulate, to $5 \mathrm{D}, g$ and $h$ for $p=0.80,0.90,0.95$, and 0.99 . They also tabulate equicoordinate and non-equicoordinate onesided percentage points for block correlation structure. Bechhofer and Dunnett [71] summarize previous tables of percentage points for equicorrelated multivariate normal and t-distributions.

\subsection{Distribution of the Wilks's Likelihood Ratio Test Statistic}

Schatzoff [72], Pillai and Gupta [73], Lee [74], and Davis [75] tabulate multiplying factors $C$ to obtain upper percentage points of the distribution of the Wilks's Likelihood Ratio Test Statistic $-[n-p-(1 / 2)$ $(m-r+1)] \log \mathrm{W}$ from the percentage points of the chi-square distribution for multivariate analysis of variance. Muirhead [76] has consolidated these into one large table. Here, $n$ is the number of multivariate measurements, $p$ is the number of regression parameter vectors, $n-p$ is the error degrees of freedom, $m$ is the dimension of multivariate measurements, and $r$ is the degrees of freedom of the general linear hypothesis. Factors for the upper $\alpha \times 100$ percent points are tabulated for $\alpha=0.100,0.050,0.025$, and 0.005 . The chi-square distribution has $m r$ degrees of freedom. The degrees of freedom $n-p-m+1$ equal 1(1)10, $10(2) 20,24,30,40,60,120$, and $\infty$. Pairs $(m, r)$ are such that $m=3(1) 10,12$, and $r \geq m$, where $r$ is up to 22 for $m=3$, and $4, r$ is up to 20 for $m=5,6$, and 7 , and $r$ is up to 18,16 , and 14 for $m=8,9$, and 10 , respectively. Pairs $(m, r)=(6,11),(6,13)$, and $(10,13)$ are excluded. For $r \leq m$ make the substitutions $m \rightarrow r, r \rightarrow m$, and $n-p \rightarrow n+r-p-m$.

\subsection{Dirichlet Distribution-Type 1}

Sobel, Uppuluri, and Frankowski [77] tabulated, to 10D, the incomplete Dirichlet integral of Type 1:

$$
\begin{aligned}
I_{p}^{(b)}(r, n) & = \\
& \frac{\Gamma(n+1)}{\Gamma^{b}(r) \Gamma(n-b r+1)} \\
& \times \int_{0}^{p} \cdots \int_{0}^{p}\left(1-\sum_{i=1}^{b} x_{i}\right)^{n-b r} \prod_{i=1}^{b} x_{i}^{r-1} \mathrm{~d} x_{i}
\end{aligned}
$$

for $p=1 / b, b=2(1) 10, r=1(1) 10$, and $n \geq b r$. This represents $\operatorname{Pr}\left\{\bigcap_{i=1}^{b}\left(X_{i} \leq p\right)\right\}$ where $X_{1}, \ldots, X_{b}$ have a joint Dirichlet distribution with the specified parameters. Also tabulated, to $10 \mathrm{D}$, are values of $I_{p}^{(b)}(r, n)$ for $p=1 / j, j=b+1(1) 10, \quad b=1(1) 10$, and 
and $r=1(1) 10$. Values of $n$ are given to, 2D, for which $I_{p}^{(b)}(r, n)=M$ for $M=0.75,0.90,0.95,0.975,0.99$, $0.999,0.9999, p=1 / j, j=b+1(1) 20, b=1(1) 10$ and $r=1(1) 10$. Additional tables are given for Generalized Stirling Numbers and for the sample size required for occupancy problems in multinomial distributions.

\subsection{Dirichlet Distribution-Type 2}

Sobel, Uppuluri, and Frankowski [78] tabulated the incomplete Dirichlet integrals of Type 2:

$$
\begin{aligned}
C_{a}^{(b)}(r, m) & =\frac{\Gamma(m+b r)}{\Gamma^{b}(r) \Gamma(m)} \\
& \times \int_{0}^{a} \cdots \int_{0}^{a} \frac{\prod_{i=1}^{b} x_{i}^{r-1} \mathrm{~d} x_{i}}{\left(1+\sum_{i=1}^{b} x_{i}\right)^{m+b r}}, \\
D_{a}^{(b)}(r, m) & =\frac{\Gamma(m+b r)}{\Gamma^{b}(r) \Gamma(m)} \\
& \times \int_{a}^{\infty} \cdots \int_{a}^{\infty} \frac{\prod_{i=1}^{b} x_{i}^{r-1} \mathrm{~d} x_{i}}{\left(1+\sum_{i=1}^{b} x_{i}\right)^{m+b r}} .
\end{aligned}
$$

The lower tail integral $C_{a}^{(b)}(r, m)$ is tabulated, to $8 \mathrm{D}$, for the parameters: $\{r=1(1) 10, b=1(1) 15, m=1(1) 15$, $a=1(1) 5$, and $\left.a^{-1}=2(1) 5\right\}$ and $\{r=m, b=1(1) 10$, $a=0.40(0.10) 0.60, \quad a=0.60(0.05) 0.80, \quad$ and $a^{-1}=$ $3(1) 10\}$.

The upper tail integral $D_{a}^{(b)}(r, m)$ is tabulated, to $8 \mathrm{D}$ or $10 \mathrm{D}$, for the parameters: $\{r=1(1) 10, b=1(1) 15$, $m=1(1) 15, a=1(1) 5$, and $\left.a^{-1}=2(1) 5\right\}, \quad\{r=m, b=$ 1(1)10, $a=3(1) 10, a^{-1}=0.40(0.10) 0.60$, and $a^{-1}=$ $0.60(0.05) 0.80\}, \quad\{m=r+1, b=1(1) 10, a=3(1) 10$, $a^{-1}=0.40(0.10) 0.60$, and $\left.a^{-1}=0.60(0.05) 0.80\right\},\{r=m$, $b=1(1) 10, a=0.40(0.10) 0.60, a=0.60(0.05) 0.80$, and $\left.a^{-1}=3(1) 10\right\},\{m=r+1, b=1(1) 10, a=40(0.10) 0.60$, $0.60(0.05) 0.80$, and $\left.a^{-1}=3(1) 10\right\}, \quad\{m=r+1, r=$ 1(1)200, $a=1$, and $b=1(1) 10\}$, and $\{m=r+2$, $r=1(1) 200, a=1$, and $b=1(1) 10\}$. Values of $a$ for which $D_{a}^{(b)}(r, m)=\delta$ are tabulated for $\delta=0.75,0.95$, $0.975,0.99,0.995,0.999, r=1(1) 50$, and $b=1(1) 10$. A table for expected waiting time in multinomial problems is also given.

\subsection{Zonal Polynomials}

Probability density functions and moments of many multivariate distributions can be evaluated using zonal polynomials. Parkhurst and James [79] tabulate zonal polynomials of order 1 through 12 in terms of sums of powers and in terms of elementary symmetric functions.

\subsection{Distributions of the Largest and Smallest Eigenvalues of Matrices of Sample Quantities}

Heck [80] charts some upper percentage points of the distribution of the largest eigenvalue of certain matrices of sample quantities from multivariate normal distribution. Edelman [81] tabulates expected values of the smallest eigenvalue of random matrices of Wishart type.

\section{Summary}

This article is a survey of the tables of probability distributions published about or after the publication in 1964 of the Handbook of Mathematical Functions, edited by Abramowitz and Stegun. The abstracts presented here have been verified from the original sources. Many of the distributions referenced here are implemented in commercial or publicly-available software systems.

\section{Acknowledgment}

The Handbook of Mathematical Functions, edited by Abramowitz and Stegun, is becoming increasingly out-of-date. A project is underway at the National Institute of Standards and Technology to develop a Web based replacement, a Digital Library of Mathematical Functions (DLMF). This survey of tables of probability distributions was done as a part of gathering background information for the DLMF. Comments by Ron Boisvert and Walter Liggett on an earlier draft have improved the paper. The following provided help with the DLMF and LATEX: Dan Lozier, Bruce Miller, Joyce Conlon, and Charles Hagwood.

\section{References}

[1] Milton Abramowitz and Irene A. Stegun, eds., Handbook of Mathematical Functions, Number 55 in Applied Mathematics Series, U.S. Department of Commerce, National Bureau of Standards, Washington, DC (1964).

[2] Egon S. Pearson and H. O. Hartley, eds., Biometrika Tables for Statisticians, Vol. I, Cambridge University Press, Cambridge, UK, third edition (1966).

[3] J. A. Greenwood and H. O. Hartley, Guide to Tables in Mathematical Statistics, Princeton University Press, Princeton, NJ (1962)

[4] D. B. Owen, Handbook of Statistical Tables, Addison-Wesley, Reading, MA (1962).

[5] Egon S. Pearson and H. O. Hartley, eds., Biometrika Tables for Statisticians, Vol. II, Cambridge University Press, Cambridge, UK (1976).

[6] Norman L. Johnson, Samuel Kotz, and A. W. Kemp, Univariate Discrete Distributions, John Wiley \& Sons, New York, NY, second edition (1992). 
[7] Norman L. Johnson, Samuel Kotz, and N. Balakrishnan, Continuous Univariate Distributions, Vol. I. John Wiley \& Sons, New York, NY, second edition (1994).

[8] Norman L. Johnson, Samuel Kotz, and N. Balakrishnan, Continuous Univariate Distributions, Vol. II. John Wiley \& Sons, New York, NY, second edition (1995).

[9] Norman L. Johnson, Samuel Kotz, and N. Balakrishnan, Discrete Multivariate Distributions, John Wiley \& Sons, New York, NY (1997).

[10] Samuel Kotz, N. Balakrishnan, and Norman L. Johnson, Continuous Multivariate Distributions, Vol. I. John Wiley \& Sons, New York, NY, second edition (2000).

[11] Sol Weintraub, Tables of the Cumulative Binomial Probability Distribution for Small Values p. Collier-Macmillan, London, UK (1963).

[12] Defense Systems Department, General Electric Company, Tables of the Individual and Cumulative Terms of Poisson Distribution, D. Von Nostrand Company, Princeton, NJ (1962).

[13] Salem H. Khamis and W. Rudert, Tables of the Incomplete Gamma Function Ratio, Justus von Liebig Verlag, Darmstadt, Germany (1965).

[14] H. Grimm, Tafeln der Negativen Binomialverteilung, Biomet. Zeit. 4, 239-262 (1962).

[15] Eric Williamson and Michael H. Bretherton, Tables of the Negative Binomial Probability Distribution, John Wiley \& Sons, New York, NY (1963).

[16] Wilburn R. Deahl, Tables of the Negative Binomial Probability Distribution for Small Values of p: A Supplement to the Tables of Williamson and Bretherton, Technical Report, Library of the University of Wyoming, Laramie, WY (1970).

[17] B. Brown, Some Tables of the Negative Binomial Probability Distribution and Their Use, RAND Corporation Memorandum, RM-4577-PR, Santa Monica, CA (1965).

[18] Gerald J. Lieberman and Donald B. Owen, Tables of the Hypergeometric Probability Distribution, Stanford University Press, Stanford, CA (1961).

[19] Ganapati P. Patil, Some Methods of Estimation for the Logarithmic Series Distribution, Biometrics 18, 68-75 (1962).

[20] Ganapati P. Patil, A. R. Kamat, and J. K.Wani, Certain Studies on the Structure and Statistics of the Logarithmic Series Distribution and Related Tables, U.S. Aerospace Research Laboratories, ARL-64-197, Wright Patterson Air Force Base, $\mathrm{OH}$ (1964).

[21] Ganapati P. Patil and J. K. Wani, Maximum Likelihood Estimation for the Complete and Truncated Logarithmic Series Distributions, Sankhya, Series A 27, 281-292 (1965).

[22] Eric Williamson and Michael H. Bretherton, Tables of the Logarithmic Series Distribution, Ann. Math. Stat. 35, 284-297 (1964).

[23] H. Grimm, Tafeln der Neyman-Verteilung Typ A, Biomet. Zeit. 6, 10-23 (1964).

[24] C. C. Sherbrooke, Discrete Compound Poisson Processes and Tables of the Geometric Poisson Distribution, Naval Research Logistics Quarterly 15, 189-203 (1968).

[25] John S. White, Tables of Normal Percentile Points, J. Am. Stat. Assoc. 65, 635-638 (1970).

[26] E. F. Fama and R. Roll, Some Properties of Symmetric Stable Distributions, J. Am. Stat. Assoc. 63, 817-836 (1968).

[27] D. R. Holt and E. L. Crow, Tables and Graphs of the Stable Probability Density Functions, J. Res. Natl. Bur. Stand., 77B, 144-198 (1973).
[28] Graham J. Worsdale, Tables of Cumulative Distribution Functions for Symmetric Stable Distributions, Applied Statistics 24, 123-131 (1975).

[29] D. B. Panton, Cumulative Distribution Function Values for Symmetric Standardized Stable Distributions, Communications in Statistics - Simulation and Computation 21, 485-492 (1992).

[30] M. T. Wasan and L. K. Roy, Tables of Inverse Gaussian Percentage Points, Technometrics 11, 591-604 (1969).

[31] M. Y. Chan, A. C. Cohen, and B. J. Whitten, The Standardized Inverse Gaussian Distribution-Tables of the Cumulative Distribution Function, Communications in Statistics-Simulation and Computation 12, 423-442 (1983).

[32] James A. Koziol, ed., CRC Handbook of Percentage Points of the Inverse Gaussian Distribution, CRC Press, Boca Raton, FL (1989).

[33] H. Leon Harter, New Tables of the Incomplete Gamma Function Ratio and of Percentage Points of the Chi-Square and Beta Distributions, U.S. Aerospace Research Laboratories, Wright-Patterson Air Force Base, OH (1964).

[34] Karl Pearson, ed., Tables of the Incomplete Gamma Function, Cambridge University Press, Cambridge, UK (1922). Reissue, 1934.

[35] H. Leon Harter, More Tables of the Incomplete Gamma Function Ratio and of Percentage Points of the Chi-Square Distribution, U.S. Aerospace Research Laboratories, WrightPatterson Air Force Base, OH (1964).

[36] Martin B. Wilk, Ram Gnanadesikan, and M. J. Huyett, Tables of Inverse Gaussian Percentage Points, Technometrics 4, 1-20 (1962).

[37] H. C. S. Thom, Direct and Inverse Tables of the Gamma Distribution. Environmental Data Service, Silver Spring, MD (1968).

[38] H. Leon Harter, A New Table of Percentage Points of the Pearson Type III Distribution, Technometrics 11, 177-187 (1969).

[39] H. Leon Harter, More Percentage Points of the Pearson Type III Distribution, Technometrics 13, 203-204 (1971).

[40] H. Leon Harter, A New Table of Percentage Points of the ChiSquare Distribution, Biometrika 51, 231-239 (1964).

[41] K. V. Mardia and P. J. Zemroch, eds., Tables of the F- and Related Distributions With Algorithms, Academic Press, New York, NY (1978).

[42] Alan Plait, The Weibull Distribution-With Tables, Industrial Quality Control 19, 17-26 (1962).

[43] E. J. Gumbel, Probability Tables for the Analysis of Extreme Value Data, Number 22 in Applied Mathematics Series, U.S. Department of Commerce, National Bureau of Standards, Washington, DC (1953).

[44] J. S. White, The Moments of Log-Weibull Order Statistics, Technometrics 11, 373-386 (1969).

[45] N. Balakrishnan and P. S. Chan, Order Statistics From Extreme Value Distribution, I: Tables of Means, Variances, and Covariances, Communications in Statistics-Simulation and Computation 21, 1199-1217 (1992).

[46] N. Balakrishnan and P. S. Chan, Extended Tables of Means, Variances, and Covariances of Order Statistics From the Extreme Value Distribution for Sample Sizes up to 30, Report, Department of Mathematics and Statistics, McMaster University, Hamilton, Canada (1992).

[47] Karl Pearson, ed., Tables of the Incomplete Beta Function, Cambridge University Press, Cambridge, UK, first edition (1934). 
[48] Karl Pearson, ed., Tables of the Incomplete Beta Function, Cambridge University Press, Cambridge, UK, second edition (1968).

[49] L. E. Vogler, Percentage Points of the Beta Distribution, Technical Note 215, National Bureau of Standards, Boulder, CO (1964).

[50] Ronald A. Fisher and Frank Yates, eds., Statistical Tables for Biological, Agricultural and Medical Research, Hafner Publishing Company Inc., New York, NY, sixth edition (1963).

[51] Fred B. Lempers and Adri S. Louter, An Extension of the Table of the Student Distribution, J. Am. Stat. Assoc. 66, 503 (1971).

[52] G. W. Hill, Reference table: "Student's" t-Distribution Quantiles to 20D, Division of Mathematical Statistics Technical Paper 35, CSIRO, Australia (1972).

[53] Norman L. Johnson, Tables of Percentile Points of Noncentral Chi-Square Distribution, Mimeo Series 568, Institute of Statistics, University of North Carolina, Chapel Hill, NC (1968).

[54] G. E. Haynam, Z. Govindarajulu, and Fred C. Leone, Tables of the Cumulative Noncentral Chi-Square Distribution, in H. L. Harter and D. B. Owen, eds., Selected Tables in Mathematical Statistics, Vol. 1, American Mathematical Society, Providence, RI (1973) pp. 1-78.

[55] Norman L. Johnson and Egon S. Pearson, Tables of Percentage Points of Noncentral Chi, Biometrika 56, 255-272 (1969).

[56] M. L. Tiku, Tables of the Power of the F-Test, J. Am. Stat. Assoc. 62, 525-539 (1967).

[57] M. L. Tiku, Doubly Noncentral F-Distribution-Tables and Applications, in H. L. Harter, D. B. Owen, and J. M. Davenport, eds., Selected Tables in Mathematical Statistics, Vol. 2, American Mathematical Society, Providence, RI (1974) pp. 139-176.

[58] S. C. Bagui, ed., CRC Handbook of Percentiles of Noncentral t-Distribution, CRC Press, Boca Raton, FL (1993).

[59] William G. Bulgren, Probability Integral of the Doubly Noncentral t-Distribution With Degrees of Freedom $n$ and Noncentrality Parameters $\delta$ and $\lambda$, in H. L. Harter, D. B. Owen, and J. M. Davenport, eds., Selected Tables in Mathematical Statistics, Vol. 2, American Mathematical Society, Providence, RI (1974) pp. 1-138.

[60] Robert E. Odeh, Critical Values of the Sample Product-Moment Correlation Coefficient in the Bivariate Normal Distribution, Communications in Statistics-Simulation and Computation 11, 1-26 (1982).

[61] Shanti S. Gupta, Probability Integrals of Multivariate Normal and Multivariate t, Ann. Math. Stat. 34, 792-828 (1963).

[62] Y. L. Tong, The Multivariate Normal Distribution, SpringerVerlag, New York, NY (1990).

[63] Harold Freeman, Arnold M. Kuzmack, and Rita Maurice, Multivariate $t$ and the Ranking Problem, Biometrika 54, 305308 (1967).

[64] Harold Freeman and Arnold M. Kuzmack, Tables of Multivariate $\mathrm{t}$ in Six and More Dimensions, Biometrika 59, 217-219 (1972).

[65] Olive J. Dunn, Richard A. Kronmal, and William A. Yee, Tables of the Multivariate t-Distribution, Technical Report, School of Public Health, University of California, Los Angeles, CA (1968).

[66] P. R. Krishnaiah, J. V. Armitage, and M. C. Breiter, Tables for the Probability Integral of the Bivariate t-Distribution, U.S. Aerospace Research Laboratories, ARL-69-0060, Wright Patterson Air Force Base, OH (1969).
[67] P. R. Krishnaiah, J. V. Armitage, and M. C. Breiter, Tables for the Bivariate $|t|$ Distribution, U.S. Aerospace Research Laboratories, ARL-69-0210, Wright Patterson Air Force Base, $\mathrm{OH}(1969)$.

[68] Yung L. Tong, On Partitioning a Set of Normal Populations by Their Location With Respect to a Control, Ann. Math. Stat. 40, 1300-1324 (1969).

[69] J. R. Trout and Bryant Chow, Table of the Percentage Points of the Trivariate t-Distribution With an Application to Uniform Confidence Bands, Technometrics 14, 855-879 (1972).

[70] John E. Dutt, On Computing the Probability Integral of a General Multivariate t, Biometrika 62, 201-205 (1975).

[71] Robert E. Bechhofer and Charles W. Dunnett, Percentage Points of Multivariate Student's t-Distributions, in R. E. Odeh, J. M. Davenport, and N. S. Pearson, eds., Selected Tables in Mathematical Statistics, Vol. 11, American Mathematical Society, Providence, RI (1988).

[72] Martin Schatzoff, Exact Distributions of Wilks's Likelihood Ratio Criterion, Biometrika 53, 347-358 (1966).

[73] K. C. S. Pillai and Arjun K. Gupta, On the Exact Distribution of Wilks's Criterion, Biometrika 56, 109-118 (1969).

[74] Y. S. Lee, Some Results on the Distribution of Wilks's Criterion, Biometrika 59, 649-664 (1972).

[75] A. W. Davis, On the Differential Equation for Meijer's $G_{p, p}^{p, 0}$ Function, and Further Tables of Wilks's Likelihood Ratio Criterion, Biometrika 66, 519-531 (1979).

[76] Robb J. Muirhead, Aspects of Multivariate Statistical Theory, John Wiley \& Sons, New York, NY (1982).

[77] Milton Sobel, V. R. R. Uppuluri, and K. Frankowski, Dirichlet Distribution-Type 1, in D. B. Owen, R. E. Odeh, and J. M. Davenport, eds., Selected Tables in Mathematical Statistics, Vol. 4, American Mathematical Society, Providence, RI (1977).

[78] Milton Sobel, V. R. R. Uppuluri, and K. Frankowski, Dirichlet Integrals of Type 2 and Their Applications, in R. E. Odeh, J. M. Davenport, and N. S. Pearson, eds., Selected Tables in Mathematical Statistics, Vol. 9, American Mathematical Society, Providence, RI (1985).

[79] A. M. Parkhurst and A. T. James, Zonal Polynomials of Order 1 Through 12, in H. L. Harter, D. B. Owen, and J. M. Davenport, eds., Selected Tables in Mathematical Statistics, Vol. 2, American Mathematical Society, Providence, RI (1974) pp. 199-388.

[80] D. L. Heck, Charts of Some Upper Percentage Points of the Distribution of the Largest Characteristic Root, Ann. Math. Stat. 31, 625-642 (1960).

[81] A. Edelman, The Distribution and Moments of the Smallest Eigenvalue of a Random Matrix of Wishart Type, Linear Algebra Appl. 159, 55-80 (1991).

About the authors: Dr. Raghu Kacker is a mathematical statistician in the Mathematical and Computational Sciences Division of the NIST Information Technology Laboratory. Dr. Ingram Olkin is professor of statistics and professor of education at the Stanford University. The National Institaute of Standards and Technology is an agency of the Technology Administration, U.S. Department of Commerce. 